PRAMANA

- journal of

physics (c) Indian Academy of Sciences

pp. $\quad 1-16$

\title{
Gravitational Collapse: The Story so far
}

\author{
Pankaj S. Joshi \\ Tata Institute of Fundamental Research, Homi Bhabha Road, Colaba, Mumbai 400005
}

\begin{abstract}
An outstanding problem in gravitation theory and relativistic astrophysics today is to understand the final outcome of an endless gravitational collapse. Such a continual collapse would take place when stars more massive than few times the mass of the sun collapse under their own gravity on exhausting their nuclear fuel. According to the general theory of relativity, this results either in a black hole, or a naked singularity- which can communicate with faraway observers in the universe. While black holes are (almost) being detected and are increasingly used to model high energy astrophysical phenomena, naked singularities have turned into a topic of active discussion, aimed at understanding their structure and implications. Recent developments here are reviewed, indicating future directions.
\end{abstract}

Keywords. gravitational collapse, black holes, naked singularities

PACS Nos 2.0

\section{Introduction}

What is the final outcome of the continual gravitational collapse of a massive star which has exhausted its nuclear fuel? While stars not very massive could stabilize as white dwarfs or neutron stars, any stellar core more massive than about five solar masses must collapse endlessly according to our present physical understanding. The question of final fate of such an endless collapse is of central importance in gravitation theory and astrophysics today. The theory to use to examine this question related to strong gravity fields is the general theory of relativity, which should be valid till the quantum gravity length scales of about $10^{-33} \mathrm{cms}$, and which would be a low energy limit of any reasonable quantum gravity theory.

In early seventies, the singularity theorems in general relativity gave some partial hints to an answer to the above question. Under a reasonable set of physical assumptions, such as causality and positivity of energy density, these theorems showed that closed trapped surfaces, which develop in gravitational collapse, give rise to spacetime singularities. Such singularities signal the onset of a phase of extreme strong gravity regions where the quantum effects should start getting important. It is only in such strong gravity fields near singularities where both general relativity and quantum gravity come into their own, and one may have an opportunity to test the effects of quantum gravity. The limitation of the singularity theorems, however, was that they only predicted the existence of singularities in collapse and cosmology, but did not give any information on their physical nature (e.g. 
how fast the densities and curvatures grow there), or the causal structure (e.g. can they communicate with faraway observers in the universe).

These questions, which are clearly vital to understanding the final fate of massive collapsing clouds, can be answered only by means of a detailed study of gravitational collapse phenomena in gravitation theory [1]. The classical spacetime singularities should be smeared out by quantum gravity, and what would really result from such an endless collapse is an extreme strong gravity region, with extreme values of physical parameters such as densities and curvatures, confined to an extraordinarily tiny region of space. If the event horizons of gravity already start developing at an earlier phase during such a collapse, the collapsing star and the eventual fireball as described above gets hidden within the horizon, disappearing from the purview of the outside observers in the universe forever. Then we have the formation of a black hole in the universe as a result of the gravitational collapse. On the other hand, if the formation of event horizon gets delayed sufficiently during the collapse, the result is the development of a naked singularity, or a visible fire ball, which can possibly send out massive radiations to faraway observers from near such strong gravity regions.

A detailed study of gravitational collapse phenomena from such a perspective has been conducted within the context of classical gravity [2]. The generic conclusions emerging from these studies are striking: While the collapse always produces curvature generated fireballs characterized by diverging curvatures and densities, trapped surfaces may not develop early enough to always shield this process from an outside observer. Specifically, as we shall show in the next section, depending on the nature of the initial data from which the collapse develops, either a black hole or a naked singularity results as the final outcome of the collapse. We then discuss several implications and generalizations of these results, giving an idea of the recent developments and future directions in this field.

\section{Spherically symmetric collapse}

Spherically symmetric collapse has been investigated from such a perspective in detail. The first studies examining the dynamical evolutions of collapsing matter clouds were due to Oppenheimer and Snyder, and Dutt [3]. As is well-known now, such a collapse of a homogeneous dust ball (the density and pressures given by $\rho=\rho(t), p=0$ ) gives rise to a black hole, where the extreme density regions are necessarily hidden from the faraway observer by the event horizon, which starts forming much earlier than the epoch of formation of singularity.

What is the outcome of the collapse, however, when the density is allowed to be inhomogeneous, which is a physically more realistic situation? The collapse of spherically symmetric inhomogeneous dust has been studied in detail, and we now know that the outcome is generically either a black hole or a naked singularity, depending on the nature of the initial data from which the collapse develops [4].

One may, however, consider dust as somewhat unrealistic form of matter, especially towards the end stages of collapse, when pressures should be important. From such a perspective, the gravitational collapse of perfect fluids, and other more general forms of matter, has been studied analytically [5] and also numerically [6]. The conclusions remain essentially the same, namely, both black holes and naked singularities do develop as end state of gravitational collapse. 
In the following, using the general treatment given by Joshi and Dwivedi [7] we show that given an arbitrary regular distribution of a general matter field at the initial epoch, there always exists an evolution from this initial data which would result either in a black hole or a naked singularity, depending on the allowed choice of free functions available in the solution. It follows that either of these objects result depending on the nature of the regular initial data from which the collapse evolves. Again the usual energy conditions ensuring the positivity of energy density and other regularity conditions will be satisfied. This method also generates wide new families of black hole solutions resulting from spherically symmetric collapse, without requiring the cosmic censorship assumption.

We consider here general type I matter fields [8], which include most of the physically important forms of matter such as dust, perfect fluids, massless scalar fields and so on. In fact, almost all observed forms of matter and equations of state are included in this general class. Our purpose is to analyze the collapse with a given initial data set such as the state of matter and the velocities of the spherical shells at the onset of collapse for a compact object, in order to determine the possibilities of this configuration evolving into either a black hole or a naked singularity. So we consider the gravitational collapse of a matter cloud that evolves from a regular initial data defined on an initial spacelike surface. The energy-momentum tensor has a compact support on this initial surface where all the physical quantities such as densities and pressures are regular and finite.

Such a matter field, in a general coordinate system, can be expressed as

$$
T^{a b}=\lambda_{1} E_{1}^{a} E_{1}^{b}+\lambda_{2} E_{2}^{a} E_{2}^{b}+\lambda_{3} E_{3}^{a} E_{3}^{b}+\lambda_{4} E_{4}^{a} E_{4}^{b}
$$

where $\left(E_{1}, E_{2}, E_{3}, E_{4}\right)$ is an orthonormal basis with $E_{4}$ and $\left(E_{1}, E_{2}, E_{3}\right)$ are timelike and spacelike eigenvectors respectively, and $\lambda_{i} \mathrm{~s}(i=1,2,3,4)$ are the eigenvalues. For such a spherically symmetric matter distribution we can choose coordinates $\left(x^{i}=t, r, \theta, \phi\right)$ adopted to this orthonormal frame, and the metric is written as,

$$
d s^{2}=-e^{2 \nu} d t^{2}+e^{2 \psi} d r^{2}+R^{2} d \Omega^{2}
$$

where $d \Omega^{2}=d \theta^{2}+\sin ^{2} \theta d \phi^{2}$ is the line element on a two-sphere. Here $\nu, \psi$ and $R$ are functions of $t$ and $r$, and the stress-energy tensor $T_{b}^{a}$ as given by equation (1) has only diagonal components in this coordinate system (i.e. we are using a comoving coordinate system), given by

$$
T_{t}^{t}=-\rho, \quad T_{r}^{r}=p_{r}, \quad T_{\theta}^{\theta}=p_{\theta}=T_{\phi}^{\phi}, \quad T_{r}^{t}=T_{t}^{r}=0
$$

The quantities $\rho, p_{r}$, and $p_{\theta}$ are the eigenvalues of $T_{b}^{a}$ and are interpreted as the density, radial pressure, and tangential stresses respectively for the cloud. We take the matter fields to satisfy the weak energy condition, i.e. the energy density as measured by any local observer must be non-negative, and so for any timelike vector $V^{a}$ we must have

$$
T_{a b} V^{a} V^{b} \geq 0
$$

which amounts to

$$
\rho \geq 0, \quad \rho+p_{r} \geq 0, \quad \rho+p_{\theta} \geq 0
$$

From the point of view of the dynamical evolution of the initial data at an epoch of time from which the collapse commences, we have a total of six arbitrary functions of $r$, namely, 


$$
\begin{array}{ll}
\nu\left(t_{i}, r\right)=\nu_{o}(r), \quad \psi\left(t_{i}, r\right)=\psi_{o}(r), \quad R\left(t_{i}, r\right)=R_{o}(r), \\
\rho\left(t_{i}, r\right)=\rho_{o}(r), \quad p_{r}\left(t_{i}, r\right)=p_{r_{o}}(r), \quad p_{\theta}\left(t_{i}, r\right)=p_{\theta_{o}}(r)
\end{array}
$$

These functions constituting the initial data are to be specified at an initial surface at an initial epoch $t=t_{i}$. The dynamical evolution of this initial data is determined by the Einstein equations, and for the metric (2) these are given by,

$$
\begin{aligned}
& T_{t}^{t}=-\rho=-\frac{F^{\prime}}{k_{o} R^{2} R^{\prime}}, \quad T_{r}^{r}=p_{r}=-\frac{\dot{F}}{k_{o} R^{2} \dot{R}} \\
& \nu^{\prime}\left(\rho+p_{r}\right)=2\left(p_{\theta}-p_{r}\right) \frac{R^{\prime}}{R}-p_{r}^{\prime} \\
& -2 \dot{R}^{\prime}+R^{\prime} \frac{\dot{G}}{G}+\dot{R} \frac{H^{\prime}}{H}=0 \\
& G-H=1-\frac{F}{R}
\end{aligned}
$$

where ( $)$ and $\left({ }^{\prime}\right)$ represent partial derivatives with respect to $t$ and $r$ respectively, $F=$ $F(t, r)$ is an arbitrary function of $t$ and $r$, and we put

$$
G=G(t, r)=e^{-2 \psi}\left(R^{\prime}\right)^{2}, \quad H=H(t, r)=e^{-2 \nu} \dot{R}^{2}
$$

The function $F(t, r)$ here is treated as the mass function for the cloud, with $F \geq 0$. In order to preserve the regularity of the initial data at $t=t_{i}$, we must have $F\left(t_{i}, 0\right)=0$, that is, the mass function vanishes at the center of the cloud.

The initial data represented by the functions $\nu_{o}, \psi_{o}, \rho_{o}, p_{r_{o}}, p_{\theta_{o}}$ and $R_{o}$ are not all independent. The equation (9), when evaluated on the initial surface, gives the relationship

$$
\nu_{o}^{\prime}\left(\rho_{o}+p_{r_{o}}\right)=2\left(p_{\theta_{o}}-p_{r_{o}}\right) \frac{R_{o}^{\prime}}{R}-p_{r_{o}}^{\prime}
$$

Furthermore, there is a coordinate freedom left in the choice of the scaling of the coordinate $r$, which can be used to reduce the number of independent initial data to four. Thus there are only four independent arbitrary functions of $r$ constituting the initial data. Evolution of this data is governed by the field equations, and we have in all five equations with seven unknowns, namely $\rho, p_{r}, p_{\theta}, \nu, \psi, R$ and $F$, giving us freedom of choice of two functions. Selection of these two free functions, subject to the given initial data and the weak energy condition above, determines the matter distribution and the metric of the spacetime and thus leads to a particular evolution for the initial data. We need to ensure the regularity of the initial data, which would be the case if the curvatures and the initial data describing the matter (the initial density and pressures) are all finite. For curvatures to be finite one must have a bounded Kretchmann scalar $K=R^{a b c d} R_{a b c d}$. A singularity will appear on the initial surface if either the density $\rho$, or one of the pressures become unbounded at any 
point on the initial surface, or $\left(F / R^{3}\right) \rightarrow \infty$ at any point. We require that on the initial surface $t=t_{i}$, the density and pressures are finite and bounded. We also have

$$
F\left(t_{i}, r\right)=\int \rho_{o}\left(R_{o}\right) R_{o}^{2} d R_{o}
$$

and hence the spacetime is singularity free initially in the sense that the Kretchmann scalar, density and pressures are all finite. But, as the collapse evolves, a singularity could develop at a later time whenever either of the density or one of the pressures become unbounded. We shall consider below such specific evolutions of the initial data which model a gravitationally collapsing matter cloud.

Using the coordinate freedom left in rescaling the radial coordinate, we rescale $r$ such that

$$
R\left(t_{i}, r\right)=R_{o}(r)=r
$$

The physical area radius $R$ then monotonically increases with the coordinate $r$, and there are no shell-crossings on the initial surface, with $R^{\prime}=1$. Since we are considering gravitational collapse, we also have $\dot{R}<0$. The initial data for a collapsing matter cloud is given in terms of the initial densities and pressures describing the initial state of matter at the onset of collapse. These are $\rho_{o}, p_{r_{o}}, p_{\theta_{o}}$, and the function $\psi_{o}$, related to the initial velocity of the collapsing shells. Next, physically reasonable matter forms may satisfy an energy condition ensuring the positivity of mass-energy densities. Therefore, at the onset of the collapse, all the initial data sets specifying the density and pressures profiles of the cloud satisfy an energy condition, and the same holds during the later evolution of collapse. Satisfying the weak energy condition implies for the initial data,

$$
\rho_{o}(r) \geq 0, \quad \rho_{o}+p_{r_{o}} \geq 0, \quad \rho_{o}(r)+p_{\theta_{o}}(r) \geq 0,
$$

and the same holds at all later epochs of collapse.

Because at the initial epoch $t=t_{i}, \nu_{0} \neq \pm \infty$, we have from Einstein equations some restrictions on the arbitrariness of the choice of the functions $\rho_{o}, p_{r_{o}}, p_{\theta_{o}}$. We have, for example,

$$
\left[p_{\theta_{o}}-p_{r_{o}}\right]_{r=0}=0
$$

For the sake of physical reasonableness, we require the center $r=0$ to be the regular center for the cloud, which means $R(t, 0)=0$. Also, one would like to have the initial density $\rho_{o}(0)>0$ at the center $r=0$. This implies that we have

$$
\rho_{o}(0)+p_{r_{o}}(0)>0, \quad \nu_{o}(r)=r^{2} h(r)
$$

where $h(r)$ is at least a $C^{1}$ function of $r$ for $r=0$, and at least a $C^{2}$ function for $r>0$. This means that the pressure gradients vanish at the center $r=0$, basically meaning that the forces vanish at the center. Here we consider this scenario for the sake of physical reasonableness, however, it is possible to give a more general formalism independent of requirements such as above.

Actually, it would be reasonable to require the pressures to be positive at the onset of the collapse, since for astrophysical bodies physically we would prefer the pressures rather than tensions. Further more, to make the scenario physically more appealing, we may 
require the density to be decreasing as we move away from the center $r=0$. In that case, for any reasonable equations of state such as $p=k \rho, 0<k<1$, (a perfect fluid), or $p=k \rho^{\gamma}$, the pressure also will decrease away from the center together with the decreasing density. This may typically be the case in the massive bodies such as stars and such other astrophysical systems. Then as such the energy conditions could impose restrictions on the maximum size of the matter cloud with such an initial density and pressure distribution. Additionally, for a compact collapsing star, at the onset of the collapse the radial stress should vanish at some boundary $r=r_{b}$ i.e. $p_{r_{o}}\left(r_{b}\right)=0$.

For the given initial data set of density and pressures there may be infinitely many collapse evolutions possible. However, we do not intend to find a particular solution of the field equations prescribing a particular evolution of any given initial data. We investigate here, within the framework of general relativity and the regular initial data to begin with, when physically allowed reasonable evolutions develop into naked or covered singularities. The answer to this question would have been simple, if we had at our disposal both an exact closed equation of state describing the state of collapsing matter, and an exact solution of the field equation. However, both of these are little understood in relativity in highly dense regions. In dust models the initial data describing the matter consists of only the initial density distribution. Dust models could be criticized due to the vanishing pressures, and it is possible that if pressures are present than the conclusions regarding the final fate of collapse could be different. We therefore look for an evolution of an arbitrary initial data set consisting of both the density and non-zero pressures, and which would reduce to dust if initial pressures are vanishing. In dust models we have that the mass function $F=F(r)$ is time independent, and $\nu=0$. We therefore consider an ansatz which is a simple extension of dust, and such that one can still incorporate initial non-vanishing pressures.

Consider the gravitational collapse of a matter cloud with a general initial data as prescribed above, and the functions $F$ and $\nu$ given as below,

$$
\nu=c(t)+\nu_{o}(R), \quad F=f(r)+F_{o}(R)
$$

From the Einstein equations, the evolution of collapse is then described by the equations,

$$
\begin{aligned}
& \nu_{o}(R)=R^{2} g(R)=\int_{0}^{R}\left(\frac{2 p_{\theta_{o}}-2 p_{r_{o}}}{r\left(\rho_{o}+p_{r_{o}}\right)}-\frac{p_{r_{o}}^{\prime}}{\rho_{o}+p_{r_{o}}}\right) d r \\
& G=b(r) e^{2 \nu_{o}} \\
& \sqrt{R} \dot{R}=-a(t) e^{\nu_{o}} \sqrt{b(r) R e^{\nu_{o}}-R+f+F_{o}} \\
& \rho=\frac{f^{\prime}}{R^{2} R^{\prime}}+\frac{F_{o}, R}{R^{2}}, \quad p_{r}=-\frac{F_{o}, R}{R^{2}} \\
& 2 p_{\theta}=R \nu, R\left(\rho+p_{r}\right)+2 p_{r}+R p_{r}, R \\
& F_{o}(R)=-\int_{0}^{R} r^{2} p_{r_{o}} d r \equiv-R^{3} p(R)
\end{aligned}
$$




$$
f(r)=\int_{0}^{r} r^{2}\left(\rho_{o}+p_{r_{o}}\right) d r \equiv r^{3} \epsilon(r)+r^{3} p(r)
$$

Here $F\left(t_{i}, r\right)=2 r^{3} \epsilon(r)$. The quantities $\epsilon(r)$ and $p(r)$ are to be treated as the average mass and pressure densities of the cloud, and are decreasing functions of $r$. Since $p_{r_{o}}$ is a positive function on the initial surface, it follows that $F_{o}<0$ throughout the spacetime, and as such the radial pressure is non-negative throughout the spacetime. The arbitrary function $b(r)$ characterizes the velocity of the spherical shells at the initial time $t=t_{i}$. We are dealing with the collapse situation with $\dot{R}<0$, therefore the arbitrary function $a(t)>0$.

We note that in the above model for evolution of the initial data set as described by the above equations, if the initial pressures vanish, i.e. $p_{r_{0}}(r)=p_{\theta_{o}}(r)=0$, we then have as the solution

$$
\begin{aligned}
& d s^{2}=-e^{a(T)} d T^{2}+\frac{R_{T}^{\prime 2}}{1+E(r)} d r^{2}+R_{T}^{2} d \Omega^{2} \\
& \nu=p_{r}=p_{\theta}=0 \quad G=b(r) \equiv 1+E(r) \quad \dot{R}_{T}=-\sqrt{E+\frac{F_{T}(r)}{R_{T}}}
\end{aligned}
$$

where we have changed the notation for $t \rightarrow T$ and $R \rightarrow R_{T}$ in order to distinguish the above solution, which is actually the Tolman-Bondi-Lemaitre solution for a dust cloud. Thus, the models here may also be viewed as directly generalizing the Tolman-BondiLemaitre models to include both the radial and tangential pressures in order to investigate the role of initial data towards the final fate of collapse.

Another important point that one has to consider is the matching of the gravitational collapse model above with a suitable exterior, which is either an asymptotically flat region, or a cosmological background. For details on this, as well as a discussion of energy conditions during the evolution of collapse, we refer to [7].

Since $r=0$ is the regular center of the cloud, meaning $R(t, 0)=0$, it follows from equation (22) that

$$
\sqrt{v} \dot{v}=-a(t) e^{\nu_{o}} \sqrt{v^{3}(h(R) b(r)-p(R))+b_{o}(r) v+\epsilon(r)+p(r)}
$$

where the arbitrary function $b(r)=1+r^{2} b_{o}(r)$, such that $b_{o}(r)$ is at least a $C^{1}$ function of $r$ for $r=0$, and a $C^{2}$ function for $r>0$, and we have introduced

$$
\begin{aligned}
& R=r v(t, r), \quad v\left(t_{i}, r\right)=1 \\
& h(R)=h(r v)=\frac{e^{2 r^{2} v^{2} g(r v)}-1}{r^{2} v^{2}}=\frac{e^{2 \nu_{0}}-1}{R^{2}}
\end{aligned}
$$

The functions $b_{o}(r), h(r v), v(t, r), g(r v)$, and $f_{o}(r)$ are all at least $C^{1}$ functions of their arguments. At $t=t_{i}$ we have $v=1$ and since $\dot{v}<0$, we have $v<1$ throughout the spacetime.

The quantity $R(t, r) \geq 0$ here is the area radius in the sense that $4 \pi R^{2}(t, r)$ gives the proper area of the mass shells at any given value of the comoving coordinate $r$ for a given 
epoch of time. The area of a shell at $r=$ const. goes to zero when $R(t, r)=0$. In this sense, the curve $t=t_{s}(r)$ such that $R\left(t_{s}, r\right)=0$ describes the singularity in the spacetime where the mass shells are collapsing to a vanishing volume, with the density and pressures diverging. This shell-focusing singularity occurs along the curve $t=t_{s}(r)$ such that $v\left(t_{s}, r\right)=0$, the Kretchmann scalar diverges at such points. Using the remaining degree of freedom left in the scaling of the time coordinate $t$ we could set $a(t)=1$. Equation (29) can then be integrated with the initial condition $v\left(t_{i}, r\right)=1$ to obtain the function $v(t, r)$. We get

$$
\int_{v}^{1} \frac{\sqrt{v} d v}{\sqrt{b_{o}(r) v e^{3 \nu_{o}}+e^{2 \nu_{o}}\left(v^{3}(h(r v)-p(r v))+\epsilon(r)+p(r)\right)}}=t
$$

where we have chosen for the sake of simplicity $t_{i}=0$. Note that the coordinate $r$ is treated as a constant in the equation. The time $t=t_{s}(r)$ that corresponds to the occurrence of singularity is then given by,

$$
t=t_{s}(r)=\int_{0}^{1} \frac{\sqrt{v} d v}{\sqrt{b_{o}(r) v e^{3 \nu_{o}}+e^{2 \nu_{o}}\left(v^{3}(h(r v)-p(r v))+\epsilon(r)+p(r)\right)}}
$$

In gravitational collapse, a singularity can also occur at $R^{\prime}=0$, which is called a shellcrossing singularity. But these are singularities of a weaker nature in general, and the spacetime can possibly be extended through these using a suitable extension procedure. However, the comoving coordinate system we use here may break down and the metric may possibly become degenerate at the points where $R^{\prime}=0$. Our purpose here is to study the shell-focusing singularity at $R=0$, which is essentially different and could be much stronger gravitationally as compared to the shell-crossings which are delta-function like singularities, caused by different shells crossing each other where the density momentarily blows up. Hence, we choose the evolution of the initial data in such a manner that any shellcrossings are avoided in the collapse, except possibly at the singularity. Here we mention that a similar situation regarding the occurrence of shell-crossings arises in Tolman-BondiLemaitre dust collapse models also. However, as has been pointed out in earlier works, for a given initial density profile one can always choose appropriate initial velocity of the dust shells such that during the evolution no shell-crossings are encountered or visa-versa.

In the present general case also, the same can be achieved by means of a suitable choice of the functions involved as specified below. At a given epoch of time, the functions $\epsilon(r)$, $p(r), \nu_{o}(R)$ and $h(R)$ are at least $C^{1}$ functions and also $\epsilon(r)$ and $p(r)$ are decreasing functions of $r$. Then $b_{o}(r)$ is an arbitrary function representing the initial velocities of the collapsing spherical shells. From equation (33) it is clear that the singularity time $t_{s}(r)$ is an explicit function of the velocity $b_{o}(r)$, which is a free function, and one can choose it in such a way that $t_{s}(r)$ is an increasing function of $r$, i.e. $d t_{s} / d r>0$. The exact nature of such velocity functions $b_{o}(r)$ for which $t_{s}(r)$ is an increasing function of $r$ depends upon the exact behavior of initial density and pressures within the cloud. For example, for a matter cloud initially satisfying an equation of state of the type $p=a \rho^{\gamma}$ one of the many possibilities is the function $b_{o}(r)>0$ such that $b_{o}^{\prime}(r)<0$ and is less than a certain minimum for $r_{b} \geq r \geq 0$. For all such functions $b_{o}(r)$, therefore, the singularity curve $t=t_{s}(r)$ is an increasing curve for all allowed values of coordinate $r$, and the successive 
spherical shells within the cloud collapse to singularity successively, and shell-crossings do not occur. Thus $R^{\prime}=v+r v^{\prime}>0$ (note that $R^{\prime}=1$ initially) throughout the spacetime. Furthermore, if $\left[r^{2} b_{o}\right]^{\prime} \geq 0$ then $\sqrt{v} R^{\prime} \leq 1$ within the cloud for $1 \geq v \geq 0$.

The central shell-focusing singularity $R=0$ occurs first at $r=0$ and the time of occurrence of such a singularity, using the above equations, is given by,

$$
t_{s_{o}}=t_{s}(0)=\int_{0}^{1} \frac{\sqrt{v} d v}{\sqrt{v^{3}\left(h_{o}-p_{o}\right)+b_{o o} v+\epsilon_{o}+p_{o}}}
$$

where $h_{o}=h(0), p_{o}=p(0), \epsilon_{o}=\epsilon(0), b_{o o}=b_{o}(0)$ are constants related to the central density and pressures.

In fact, near the center $r=0$ we have

$$
t_{s}(r)=t_{s_{o}}+r X(0)+O\left(r^{2}\right) \ldots
$$

where the function $X=X(0)$ is given by

$$
X(0)=\int_{0}^{1} \frac{\sqrt{v}\left(\epsilon_{1}+p_{1}+b_{1} v-v^{4} h_{1}\right) d v}{\left(v^{3}\left(h_{o}-p_{o}\right)+b_{o o} v+\epsilon_{o}+p_{o}\right)^{3 / 2}}
$$

where $\epsilon_{1}=-\epsilon^{\prime}(0), p_{1}=-p^{\prime}(0), b_{1}=-b_{o}^{\prime}(0), h_{1}=h,_{R}(0)$.

For the case we have been considering, where pressures have been taken to be positive, the central singularity at $r=0$ could be naked, but all subsequent singularities with $r>0$ are covered as the quantity $F / R \rightarrow \infty$ and the trapped surfaces and the apparent horizon develop prior to the formation of the singularity. We note that when the pressures are allowed to be negative, still subject to the validity of the weak energy condition, the other parts of the singularity can be visible in principle [9]. It thus remains to examine only the nature of the central singularity.

Within the collapsing cloud the apparent horizon is given by the condition $R / F=1$. It is the boundary of the trapped surface region in the spacetime. The behavior of the apparent horizon curve (which meets the central singularity at $R=r=0$ ) near the center essentially determines the visibility, or otherwise, of the central singularity. For example, it is known within the context of the Tolman-Bondi-Lemaitre models that the apparent horizon is either past pointing timelike or null, or it can be spacelike, as is seen by examining the nature of the induced metric on this surface. This is unlike the event horizon which is always future pointing null. If the neighborhood of the center gets trapped earlier than the singularity, then it is covered, and if that is not the case the singularity can be naked, with families of nonspacelike trajectories escaping from it.

To examine the existence or otherwise of such families, and to examine the nature of the central singularity occurring at $R=0, r=0$ in the general class of models being considered here, let us consider the equation of the outgoing radial null geodesics which is given by,

$$
\frac{d t}{d r}=e^{\psi-\nu}
$$

The singularity appears at the point $v\left(t_{s}, r\right)=0$, which corresponds to $R\left(t_{s}, r\right)=0$. Hence, if there are future directed outgoing radial null geodesics, terminating in the past 
at the singularity, then along these trajectories we have $R \rightarrow 0$ as $t \rightarrow t_{s}$. Writing the equation for these radial null geodesics in terms of the variables $\left(u=r^{5 / 3}, R\right)$ we obtain,

$$
\frac{d R}{d u}=\frac{3}{5}\left(\frac{R}{u}+\frac{\sqrt{v} v^{\prime}}{(R / u)^{1 / 2}}\right) \frac{1-\frac{F}{R}}{\sqrt{G}(\sqrt{G}+\sqrt{H})}
$$

If the null geodesics terminate in the past at the singularity with a definite tangent, then at the singularity the tangent to the geodesics $d R / d u>0$ in the $(u, R)$ plane, and must have a finite value. In the case of collapsing matter cloud we are considering, all singularities at $r>0$ are covered since $F / R \rightarrow \infty$, and therefore $d R / d u \rightarrow-\infty$. So only the singularity at center $r=0$ could be naked. As mentioned earlier, for the case when $R^{\prime}>0$ near the central singularity, we have

$$
x_{o}=\lim _{t \rightarrow t_{s}, r \rightarrow 0} \frac{R}{u}=\lim _{t \rightarrow t_{s}, r \rightarrow 0} \frac{d R}{d u} \Rightarrow x_{o}^{3 / 2}=\frac{3}{2} X(0)
$$

where $X(0)$ is given by equation (36). Because $X(0)>0$ the singularity is at least locally naked. The behavior of outgoing radial null geodesics in the neighborhood of the singularity are described by $R=x_{o} u$ in $(R, u)$ plane and in $(t, r)$ plane it is given by

$$
t-t_{s}(0)=x_{o} r^{5 / 3}
$$

One can also write the equation for these radial null geodesics in terms of the variables $(t, R)$ in order to see how the area radius $R$ grows along these outgoing null geodesics with increasing values of time. As mentioned earlier, for the case where $R^{\prime}>0$ near the central singularity, we get

$$
X_{o}=\lim _{t \rightarrow t_{s}, r \rightarrow 0} \frac{R}{t-t_{s}(0)}=\lim _{t \rightarrow t_{s}, r \rightarrow 0} \frac{d R}{d t}==\lim _{t \rightarrow t_{s}, r \rightarrow 0}\left[e^{\nu} \frac{1-\frac{F}{R}}{\sqrt{G}+\sqrt{H}}\right]=1
$$

Again this shows that the singularity is naked at least locally. In fact, as pointed out above, it follows that for $b_{o}^{\prime}(0) \neq 0$ the area coordinate behaves as $R=$ const. $\times r^{5 / 3}$ near the singularity.

The global visibility of such a singularity, which is locally naked as above, will depend on the overall behavior of the various functions concerned within the matter cloud and we shall not go into those details presently. It has been seen, however, from the study of various examples so far, that once the singularity is locally naked, one can almost always make it globally visible by a suitable choice of allowed functions. Note that in cases where the choice of $b_{o}(r)$ is such that $X(0)<0$ the singularity would be covered. When $X(0)=0$, one has to consider the next higher order expansion term which is nonvanishing in the equation (35), and that will then determine the nature of the singularity by essentially a similar analysis.

\section{Open issues}

It may be fair to conclude, as pointed out above, that generically the gravitational collapse of a massive matter cloud would produce either a black hole or a naked singularity as 
the final state, depending on the nature of the initial data developing the collapse. The latter would essentially consist of densities and velocities profiles, and the velocities of the collapsing shells. Such models respect the physical reasonableness requirements such as positivity of energy densities, and regularity of initial data.

Such a scenario gives rise to several important questions and open problems, some of which are stated below. While our discussion may not be exhaustive, we hope this will point to some future directions in this area.

\subsection{How to formulate the cosmic censorship conjecture?}

Actually, it was this very important and basic question which led me into my studies on gravitational collapse. Since there was no rigorous formulation available for this hypothesis, not to speak of a proof, what was really needed I thought was a detailed and deeper study of the collapse phenomena in gravitation theory. It does not appear we are any closer to an answer still.

What has happened, however, is we know now that many of the possibilities suggested earlier towards formulating this conjecture do not work. Various past suggestions included, for example, that naked singularities will not occur when energy conditions are obeyed, or even if they occur they will be gravitationally weak and removable, or no naked singularities occur when we allow for pressures, or when we use a reasonable form of matter and equation of state, or that in realistic cases only a zero measure set of photon and particle trajectories come out, and so on. As pointed out above, naked singularities do occur even when we impose such 'reasonable' conditions. The real issue then, in the light of our current knowledge of gravitational collapse phenomena, is the genericity and stability of these objects in gravitation theory. Thus, a formulation I propose is:

No naked singularities forming in gravitational collapse of reasonable matter fields, developing from regular initial conditions, can be generic or stable.

Of course, one would need to define and formulate these concepts of 'genericity' and 'stability' in gravitation theory in a much more precise manner, and that is no easy task which may require sophisticated mathematical tools. We describe some attempts in that direction below. Then, trying to prove such a version will be the next challenge. Finally, if cosmic censorship fails in classical gravity, it is possible that quantum gravity may provide a hope, restoring some version of a censorship in the universe.

\subsection{Do naked singularities occur in non-spherical collapse?}

The spherically symmetric collapse has been studied extensively, as discussed above. It is then important to know if the conclusions derived in this case hold for non-spherical collapse as well. This issue is largely open, as there are no good models available presently for studies of this kind.

Some indicative studies are available, however, which tell us that naked singularities are not necessarily ruled out as soon as we go away from sphericity [10]. Shapiro and Teukolsky studied oblate and prolate collapsing configurations, and Nakamura and others studied spindle and cylindrical naked singularities. Also, Barabes and Israel made an analytical study of non-spherical collapse. The quasi-spherical collapse models due to Szekeres were 
studied by Joshi and Krolak, who found the nature of naked singularities developing in this case to be very similar to the dust collapse situation. Clearly, more remains to be done here, and numerical models may be of help while studying non-spherical collapse.

\subsection{Are they stable and generic?}

We characterized here wide new families of black hole and naked singularity solutions forming in spherical collapse in terms of the evolutions of the initial data for the collapsing object. What we still do not know is the actual measure of each of these classes in the space of all possible evolutions allowed from a given general and arbitrary but physically reasonable initial data. This is related closely to the issue of stability of naked singularities. As is well-known, the stability in general relativity is a complicated issue because there is no well-defined formulation or criteria to test stability. Fast evolving numerical codes for core collapse models may possibly provide further insights here. All the same, these classes appear to be generically arising in the collapse models considered here, at least within spherical symmetry, in that they are not an isolated phenomena but belong to a general family. Because, given any density and pressure profiles for the cloud, there exists an evolution which will lead to either a black hole or a naked singularity as desired, as the end product of collapse. Also, while discussing stability and genericity, one has to be careful on the criterion one used to test the same, because sometimes a criterion is used which makes black holes also unstable while trying to show the instability of naked singularities.

Given the complexity of the field equations, if a phenomenon occurs so widely in spherical symmetry, it is not unlikely that the same would be repeated in more general situations. In fact, before the advent of singularity theorems, it was widely believed that the singularities found in more symmetric situations will go away once we go to general enough spacetimes.

The massless scalar field collapse has been studies in detail from such a perspective of genericity, analytically by Christodoulou [11], and numerically by Choptuik and others [12]. In particular, Christodoulou showed that globally naked singularities are non-generic for the case of massless scalar field collapse. In numerical studies, the collapse was studied for a generic, smooth, one-parameter family of initial data. There is a critical value for the parameter concerned which produces a critical solution which has a naked singularity.

An important indicator in this connection is the imploding Vaidya model, where the singularity is naked when the mass parameter $\lambda \leq 1 / 8$, and a black hole develops for $\lambda>1 / 8$. The parameter $\lambda$ represents here the initial data in the form of the rate of mass loss. Thus, the singularities, both naked and covered, are stable against the perturbation of the parameter $\lambda$, and the point $\lambda=1 / 8$ is the critical point indicating the transition from one phase to the other (see e.g. [2] for details).

A similar situation arises in the case of dust collapse also where the perturbation in the initial density or velocity distribution within a certain domain does not alter the nature of the singularity. The analysis here in general has a significance in that the nature of the singularity is seen to be stable in a certain sense against the perturbation of the initial data. It is possible in this case to consider mathematical structures on the initial data space to examine stability issues more rigorously [13]. Further, Iguchi, Nakao, and Harada [14] examined the stability of dust collapse models against odd-parity perturbations, which 
correspond to rotational motion of the dust fluid. Their results indicate that the naked singularity formation process appears to be stable against such metric perturbations.

In fact, it was pointed out recently by Mena, Tavakol, and Joshi [15], who considered the fully general class of all possible density profiles at the initial epoch from which the dust collapse develops, that naked singularities may be unstable in such a fully general context. However, they showed that when one considers the class of physically motivated density profiles with density higher at the center, and decreasing away from the center, then the naked singularity formation stabilizes. This is a potentially important point to bear in mind in general debates regarding the stability and genericity of naked singularities in gravitational collapse.

\subsection{What are the basic properties and structure of naked singularities?}

In order to have a better insight into problems such as above related to genericity and stability, it would be important to understand better the structure and basic properties of naked singularities. The essential features that emerge from the study of gravitational collapse, as described in the previous section, towards the structure of naked singularity are the following:

(a) There is an existence of a naked singularity, in terms of a range available in the parameter space.

(b) There exists a non-zero measure set of families of non-spacelike trajectories- photons as well as particles world lines coming out, as opposed to isolated trajectories.

(c) This turns out to be a powerfully strong curvature singularity, where the curvature is evaluated in the limit of approach to the singularity.

The relevance of this last point is that in such a case it would not be possible to extend the spacetime through such a singularity, and it would be an unavoidable feature of the spacetime. We refer to [16] for further details on aspects of strength of singularities. In fact, these features are common to the collapse models including radiation collapse, dust, perfect fluids, self-similar as well as non-self-similar collapse models.

One gets a better insight into the structure and properties of these objects by examining exact models. One such class which has been examined in detail is that with non-zero tangential pressures, but where the radial pressure vanishes [17]. Again both naked singularities and black holes form here but several interesting properties of naked singularities become clearer.

Much insight into the collapse phenomena has been gained by studies of self-similar collapse models. The advantage here is, because of the geometrical symmetry, a complete integration of the photon and particle trajectories is possible, and many interesting features come out. For an excellent recent review see [18].

\subsection{What role do the quantum effects play?}

A question frequently asked is: Are singularities, naked or covered, relevant at all- quantum gravity must wash them away. But this is missing the actual issue. One certainly hopes that in a suitable quantum gravity theory the singularities will be smeared out. However, the issue is whether the extreme strong gravity regions formed due to gravitational collapse 
are visible to faraway observers or not. Because collapse certainly proceeds classically till the quantum gravity starts governing the situation at the scale of Planck length or so, that is, till the extreme gravity configurations have developed due to collapse. And it is the visibility or otherwise of such regions that one is discussing.

The point is, classical gravity implies existence of strong gravity regions, where both classical and quantum gravity come into their own. In fact, as pointed out by Wald (see Ref. [1]), if naked singularities develop, then in a literal sense we come face-to-face with the laws of quantum gravity whenever gravitational collapse to such an event occurs in distant regions of our universe. Thus, collapse phenomena may provide us with a possibility of actually testing quantum gravity laws.

From such a perspective, many studies have been conducted on quantum effects near naked singularities [19]. In particular, Vaz and Witten worked out the spectrum of quantum radiation from a naked singularity, in analogy to the Hawking radiation from black holes. It is possible that quantum effects near the naked singularities may help us restore some kind of a quantum cosmic censorship, or these quantum effects could give rise to interesting signatures for naked singularities.

\subsection{What possible astrophysical implications they may have?}

In the case of occurrence of naked singularity configurations developing in gravitational collapse, the emissions of light or particles from the ultra-dense regions, i.e. the fireballs, are possible to an outside observer in the universe. Would this have observational consequences? From such a perspective, several works have examined the possible astrophysical implications of what happens when collapse of a massive star results into a naked singularity, rather than a black hole.

It was examined recently if a naked singularity could be a good candidate for a strong source of gravity waves [14]. The frequency range at which naked singularities may radiate gravity waves was estimated by Thorne [20]. Various observational possibilities to detect cosmic censorship violations have been suggested recently by Krolak [21].

Both classical as well as quantum effects in the vicinity of such a visible fireball may combine to produce observable signatures for a faraway observer in the universe. Such a possibility was explored recently by Joshi, Dadhich, and Maartens [22] in connection with the gamma rays bursts which remain one of the most intriguing puzzles in astronomy. While collapse always produces the fireball with diverging curvatures and densities, late formation of trapped surfaces may allow this mostly radiation dominated fireball to expand and create shocks in the surrounding medium. In this sense, such collapse generated fireballs could provide natural candidates for a central engine required for the production of gamma rays bursts.

\section{Conclusion}

It appears from considerations such as above that the occurrence of singularities, naked or otherwise, is inherent in the theory of general relativity, and a distinction between these cases may not be possible through general relativity alone. 
In fact, the investigations on gravitational collapse phenomena in gravitation theory seem to have generated by now a somewhat general consensus, that both black holes and naked singularities do develop as a result of continual gravitational collapse. The basic question remaining is that regarding the genericity and stability of naked singularities, whenever they arise in a realistic collapse, as we discussed here. These are not, however, the questions easy to conclude as there is no standard and unique definition of genericity and stability available in gravitation theory.

Under the situation, while efforts are continuing to develop such concepts in more precise and better manner, naturally many studies have also attempted a better understanding of the nature and structure of naked singularities, and have tried to investigate their astrophysical implications, if any. Such a scenario has turned this into a field of quite an active discussion and interest, even to the extent of attracting articles in the popular press [23].

\section{Acknowledgements}

It is my pleasure to acknowledge interesting comments and useful discussions from Jiri Bicak, Naresh Dadhich, Roy Maartens, Sunil Maharaj, Ramesh Narayan, J. V. Narlikar, N. Panchapakesan, Reza Tavakol, A. K. Raychaudhuri, P. C. Vaidya, and several other friends at ICGC2000.

\section{References}

[1] For some recent reviews see e.g. R. M. Wald, gr-qc/9710068; R. Penrose, in Black holes and relativistic stars, ed. R. M. Wald, University of Chicago press (1998); P. S. Joshi, in Singularities, black holes and cosmic censorship, IUCAA Publications, Pune (1997) (gr-qc/9702036); A. Krolak, gr-qc/9910108; T. P. Singh, gr-qc/9805066; S. Jhingan and G. Magli, gr-qc/9903103.

[2] P. S. Joshi, 'Global aspects in gravitation and cosmology', Clarendon Press, OUP, Oxford (1993).

[3] J. R. Oppenheimer and H. Snyder, Phys. Rev. 56, p.455 (1939); B. Datt, Z. Physik 108, p.314 (1938).

[4] D. M. Eardley and L. Smarr, Phys. Rev. D 19, (1979) p.2239; D. Christodoulou, Commun. Math. Phys. 93 (1984) p. 171; R. P. A. C. Newman, Class. Quantum Grav. 3 (1986) p.527; B. Waugh and K. Lake, Phys. Rev. D 38 (1988) p.1315; I. H. Dwivedi and P. S. Joshi, Class. Quant.Grav., 9, L69 (1992); P. S. Joshi and I.H. Dwivedi, Phys. Rev. D47, p.5357 (1993); P. S. Joshi and T. P. Singh, Phys. Rev. D51, p.6778 (1995); T. P. Singh and P. S. Joshi, Class. Quantum Grav. 13, p.559 (1996); I. H. Dwivedi and P. S. Joshi, Class. Quant. Grav. 14, p.1223 (1997); S. Jhingan and P. S. Joshi, Ann. of Isr. Phys. Soc., 13, p.357 (1998).

[5] P. S. Joshi and I. H. Dwivedi, Commun. Math. Phys. 146, p.333 (1992); Lett. Math. Phys. 27, p. 235 (1993); Commun. Math. Phys. 166, p.117 (1994); Class. Quantum Grav. 16, p.41 (1999); K. Lake, Phys. Rev. Lett. 68, p.3129 (1992).

[6] A. Ori and T. Piran, Phys. Rev. Lett. 59, p.2137 (1987); ibid., Phys. Rev. D 42, p.1068 (1990); T. Harada, Phys. Rev. D58, p.104015 (1998).

[7] P. S. Joshi and I. H. Dwivedi, Class. Quantum Grav. 16, p.41 (1999).

[8] S. W. Hawking and G. F. R. Ellis, The large scale structure of space-time, Cambridge University Press, Cambridge (1973).

[9] Cooperstock F., Jhingan S., Joshi P. S. and Singh T. P., Class. Quantum Grav. 14, p.2195 (1997).

[10] S. L. Shapiro and S. A. Teukolsky, Phys. Rev. Lett. 66, p.994 (1991); ibid., Phys. Rev. D45, p.2006 (1992); P. S. Joshi and A. Krolak, Class. Quant. Grav. 13, p. 3069 (1996); T. Nakamura, M. shibata and K. Nakao, Prog. Theor. Phys. 89, p.821(1993). 
[11] D. Christodoulou, Ann. of Math. 149, p.183 (1999); 140, p.607 (1994); M. Roberts, Gen. Relat. Grav. 21, p.907 (1989).

[12] M. W. Choptuik, Phys. Rev. Lett. 70, p.9 (1993); see C. Gundlach, gr-qc/0001046, and references therein.

[13] R. V. Saraykar and S. H. Ghate, Class. Quantum Grav. 16, p.281 (1999).

[14] H. Iguchi, K. Nakao and T. Harada, Phys. Rev. D57, p.7262 (1998); H. Iguchi, T. Harada and K. Nakao, Prog. Theor. Phys., 101, p.1235 (1999); ibid., 103, p.53 (2000).

[15] F. Mena, R. K. Tavakol and P. S. Joshi, gr-qc/0002062.

[16] S. S. Deshingkar, P. S. Joshi and I. H. Dwivedi, Phys. Rev D59, p.044018 (1999); B. C. Nolan, gr-qc/0001026.

[17] G. Magli, Class. Quant. Grav. 14, p.1937 (1997); G. Magli, gr-qc/9711082; T. Harada, K. Nakao and H. Iguchi, Class. Quantum Grav. 16, p.2785 (1999); S. Jhingan and G. Magli, grqc/9902041; S. Barve, T. P. Singh and L. Witten, Gen. Relat. Grav. 32, p.697 (2000).

[18] B. J. Carr and A. Coley, Class. Quantum Grav. 16, R31 (1999).

[19] L. Ford and L. Parker, Phys. Rev. D17 (1978) 1485; W. A. Hiscock, L. G. Williams and D. M. Eardley, Phys. Rev. D26 (1982) 751; C. Vaz and L. Witten, Phys. Letts. B325 (1994) 27; Class. Quant. Grav. 12 (1995) 1; ibid. 13 (1996) L59; Nucl. Phys. B487 (1997) 409; gr-qc/9804001; S. Barve, T. P. Singh, C. Vaz and L. Witten, Nucl. Phys. B 532, p.361 (1998); T. Harada, H. Iguchi and K. Nakao, Phys. Rev. D61, p.101502 (2000); ibid., gr-qc/0005114.

[20] K. S. Thorne, in Relativistic Astrophysics, eds. B. J. T. Jones and D. Markovic, Cambridge University Press (1997).

[21] A. Krolak, gr-qc/9910108.

[22] P. S. Joshi, N. Dadhich, and R. Maartens, (gr-qc/0005080) Mod. Phys. Lett. A, 15, p.991 (2000); S. K. Chakravarti and P. S. Joshi, (hep-th/9208060) Int. J. Mod. Phys. D3, 647 (1994); T. P. Singh, gr-qc/9805062; E. Witten, in Quantum teory and beyond, eds. F. Mansouri and J. Sciano (1992).

[23] M. W. Browne, New York Times, Feb 12, 1997. 